\title{
Positive airway pressure therapy for sleep-disordered breathing confers short-term benefits to patients with spinal cord injury despite widely ranging patterns of use
}

\author{
Jeanette P. Brown ${ }^{1} \cdot$ Kristy A. Bauman ${ }^{1} \cdot$ Armando Kurili $^{1} \cdot$ Gianna M. Rodriguez ${ }^{2} \cdot$ Anthony E. Chiodo $^{2}$. \\ Robert G. Sitrin ${ }^{1} \cdot$ Helena M. Schotland ${ }^{1,3}$
}

Received: 3 October 2017 / Revised: 27 December 2017 / Accepted: 5 February 2018 / Published online: 7 March 2018

(c) International Spinal Cord Society 2018

\begin{abstract}
Study design Prospective, cohort study.

Objectives To evaluate the effectiveness of bi-level positive airway pressure (PAP) therapy and the patterns of use for sleepdisordered breathing (SDB) in individuals with spinal cord injury (SCI).

Setting Academic tertiary care center, USA.

Methods Overall, 91 adults with C1-T6 SCI for $\geq 3$ months were recruited and 74 remained in the study to be evaluated for SDB and follow-up. Individuals with SDB but no nocturnal hypercapnia (NH) were prescribed auto-titrating PAP. Those with NH were prescribed PAP with volume-assured pressure support. Device downloads and overnight transcutaneous capnography were performed at 3, 6, and 12 months to quantify PAP use and effectiveness. Participants kept daily event logs, and quality of life (QOL) questionnaires were performed after 3, 6, and 12 months.

Results Overall, $45 \%$ of 91 participants completed the study. There was great diversity among SCI patients in PAP utilization; after 3 months, $37.8 \%$ of participants used PAP for $\geq 70 \%$ nights and $\geq 240$ min per night, whereas $42.2 \%$ seldom used PAP and $20 \%$ used PAP sporadically or for short periods. PAP therapy was effective in improving OSA in $89 \%$ and nocturnal hypercapnia in 77\%. Higher PAP pressures predicted higher levels of device use. There were marked reductions in symptoms of autonomic dysreflexia (AD) and orthostatic hypotension as well as some improved indices of QOL.

Conclusions Despite widely diverse patterns of use, PAP therapy may have short-term benefits with regard to QOL and reducing episodes of dizziness and autonomic dysreflexia.
\end{abstract}

Electronic supplementary material The online version of this article (https://doi.org/10.1038/s41393-018-0077-z) contains supplementary material, which is available to authorized users.

$\triangle$ Jeanette P. Brown

jeanette.brown@hsc.utah.edu

1 Pulmonary and Critical Care Medicine Division, Department of Internal Medicine, University of Michigan Health System, Ann Arbor, MI 48109, USA

2 Department of Physical Medicine and Rehabilitation, University of Michigan Health System, Ann Arbor, MI 48109, USA

3 Department of Neurology, Sleep Disorders Center, University of Michigan Health System, Ann Arbor, MI 48109, USA

\section{Introduction}

Obstructive sleep apnea (OSA) is very common in individuals with spinal cord injury (SCI), with studies almost uniformly finding a prevalence of $40-80 \%$ [1-6]. This prevalence is remarkably high relative to the $2-9 \%$ reported for healthy participants [7, 8]. Other forms of sleepdisordered breathing (SDB) in SCI may include central sleep apnea (CSA) and nocturnal hypoventilation (NH) with an estimated prevalence of up to $60 \%$ and $28 \%$, respectively $[1,9]$. The level of motor deficit, neck circumference, abdominal girth, respiratory muscle weakness, reduced ventilatory drive during sleep, analgesics, and other sedating medications may be contribute to SDB after SCI, but they have not been shown consistently to do so $[1,2,5]$.

In the general population, OSA is associated with increased risk for myocardial infarction, stroke, congestive heart failure, and the "metabolic syndrome" (increased 
Fig. 1 Flow diagram of study protocol, treatment stratification, and numbers of study participants at each time point

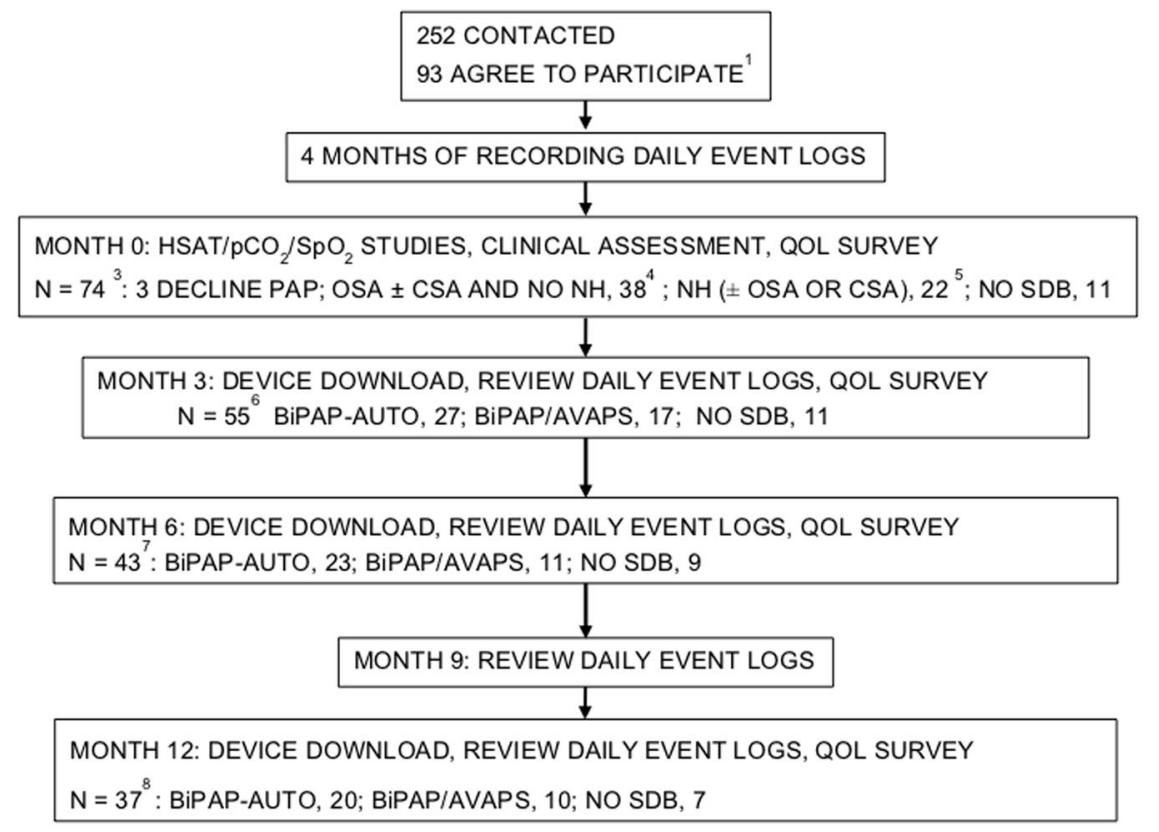

1. 2 SUBSEQUENTLY FOUND TO BE INELIGIBLE

2. $\mathrm{HSAT} / \mathrm{pCO}_{2} / \mathrm{SpO}_{2}$ RESULTS ARE DETAILED IN REFERENCE 1

3. 3 PRESCRIBED BUT REFUSED DEVICE (2 BIPAP-AUTO, 1 BIPAP-AVAPS) AND REMAINED IN STUDY 17 DECLINED FURTHER PARTICIPATION OR UNABLE TO COMPLETE STUDIES AT MO 0 (INCL. 2 DEATHS) 4. PRESCRIBED AUTO-TITRATING BILEVEL PAP (BIPAP-AUTO)

5. PRESCRIBED AVERAGE VOLUME-ASSURED PRESSURE SUPPORT (BIPAP-AVAPS)

6. 16 WITHDREW FROM STUDY DURING MO 0-3 INTERVAL

7. 12 WITHDREW FROM STUDY DURING Mo 3-6 INTERVAL

8. 6 WITHDREW FROM STUDY DURING MO 6-12 INTERVAL

visceral fat, hypertension, glucose intolerance, and hyperlipidemia) [10-17]. We know very little about the adverse clinical consequences of SDB in individuals with SCI [18]. Standard treatment of SDB is provided by non-invasive positive airway pressure (PAP) therapy. In 2009, the Centers for Medicare \& Medicaid Services (CMS) adopted a requirement of $4 \mathrm{~h}$ of PAP use on $70 \%$ of nights, or 21 days in a consecutive 30-day period, to continue medical coverage [19]. Adherence with use of PAP devices is typically low in the general population, with most studies demonstrating near $50 \%$ long-term compliance, and rates rarely as high as $80 \%$ [19-21]. Factors associated with low adherence include low socioeconomic status, claustrophobia, post-traumatic stress disorder, PAP side effects such as air leakage, skin abrasion, and mask discomfort, nasal congestion, dry throat, and frequent awakenings [22-25]. The mode of PAP delivery such as CPAP, auto-titrating PAP, and bi-level PAP may also influence adherence [26-28].

There are few studies, generally based on patient reports, addressing treatment of SDB in individuals with SCI [18, 29]. A retrospective review of participants with SCI and sleep apnea found that only $43 \%$ were receiving PAP treatment and the majority of those not receiving treatment had been intolerant of PAP or had refused treatment. The most common reason cited for PAP intolerance was mask discomfort. In addition, there was a significantly lower adherence rate in patients with high-level motor complete injuries [18]. A prospective study of auto-titrating CPAP in 14 individuals with acute SCI and OSA found an adherence rate of $50 \%$ and an improvement in sleepiness over 3 months of treatment [29]. Health-related quality of life did not seem to be greatly affected by CPAP treatment [29]. It is likely that for SCI patients, sleep is often disturbed not only by SDB, but also by other factors such as nocturnal care requirements, pain, and spasticity. This raises the possibility that factors determining adherence to SDB treatment may be entirely distinct from the general population. Similarly, the complex comorbidities of the SCI patient may affect the benefits of SDB treatment.

The goals of the present study were to prospectively evaluate adherence with PAP therapy in individuals with SCI and SDB using data recorded by the PAP device, rather than relying on patients' histories or survey data. Factors that might influence adherence such as mask type, device settings, and level of SCI were assessed. In addition, we examined whether treatment of SDB may influence common comorbidities of SCI. In obstructive sleep apnea, it has been observed that SDB induces sustained elevation in catecholamine levels and is also associated with sympathetic hyperactivity with reduced response to alpha and beta 
receptor agonists, as reviewed in Bisogni et al. [30]. Accordingly, we hypothesized that treating SDB could have a positive effect on autonomic dysreflexia and orthostatic hypotension. Lastly, we sought to determine whether treatment of SDB influences quality of life indices.

\section{Methods}

Permission was provided by the University of Michigan Institutional Review Board, project HUM00051504. The study was performed between March 2012 and December 2015. Eligibility was determined by reviewing the University of Michigan SCI Model System database, which includes $>90 \%$ of SCI patients in the region. Participants were eligible if $\geq 18$ years old, with C1-T6 spinal cord injury of $\geq 3$ months' duration, and lived within 100 miles of the study site. Exclusion criteria included: inability to provide informed consent, comorbid condition that limited life expectancy to $\leq 1$ year, active duty military personnel, ventilator-dependence, established diagnosis of SDB or prior use of non-invasive positive pressure ventilation, except during a hospitalization $\geq 3$ months prior to enrollment.

At enrollment, participants were provided daily event logs to record episodes with symptoms of autonomic dysfunction, respiratory infections, and episodes of mucus plugging/atelectasis to be completed for a 4-month observation period, and for 1 year after initiation of the study (Appendix Table 3). At the initiation of the study period (month 0), height, weight, blood pressure, waist circumference, neck circumference and body mass index (BMI) were measured, and medical records were reviewed. Participants were compensated with $\$ 50$ at month 0 and every 3 months thereafter. Motor level and completeness of SCI were determined according to the International Standards for the Neurological Classification of Spinal Cord Injury [31]. At month 0, SF-12v.2, Brief Pain Inventory-SF (BPI) and Epworth Sleepiness Scale (ESS) questionnaires (Appendix Table 4) were administered. Quality of life questionnaires and device downloads were performed at 3, 6, and 12 months. Event logs were collected every 3 months for the year of follow-up. Figure 1 details the study protocol.

At month 0, SDB was assessed in participants' homes with a home sleep apnea test (HSAT) combined with overnight oxygen saturation $\left(\mathrm{SpO}_{2}\right) /$ transcutaneous $\mathrm{pCO}_{2}$ (tc- $\mathrm{pCO}_{2}$ ) monitoring as previously described in detail [1]. The HSAT was performed with a Stardust II system Type III portable PSG system (Phillips Respironics, Murrysville, PA) incorporating an airflow sensor, pulse oximeter, and a single chest/abdominal belt to detect respiratory effort. Transcutaneous $\mathrm{pCO}_{2}$ monitoring was performed with a
SenTec Digital Monitor (SenTec, Therwil, Switzerland). On the day of testing, the study coordinator (AK) went to the participant's home with calibrated equipment and instructed participants and caregivers on proper lead placement, and starting recording at lights-out and stopping recording on awakening in the morning. The data were downloaded to the manufacturer's software and the automated analysis was reviewed and re-scored manually by a physician board certified in sleep medicine (HMS). Participants diagnosed with nocturnal hypercapnia were prescribed bi-level positive airway pressure-average volume-assured pressure support (BiPAP-AVAPS; Respironics, Murraysville, PA). Initial settings were: respiratory rate $10-12$, expiratory PAP (EPAP) $6 \mathrm{cmH}_{2} \mathrm{O}$, inspiratory PAP (IPAP) minimum 12 $\mathrm{cmH}_{2} \mathrm{O}$ and maximum $25 \mathrm{cmH}_{2} \mathrm{O}$, target tidal volume 8 $\mathrm{mL} / \mathrm{kg}$. This device maintains the programmed EPAP and auto-titrates the IPAP to achieve the target average tidal volume; other setting adjustments were made as needed for patient comfort and effectiveness. Individuals with SDB but no hypercapnia were started on bi-level positive airway pressure-Auto (BiPAP-Auto; Respironics). Initial settings were EPAP minimum $5 \mathrm{cmH}_{2} \mathrm{O}$ and maximum $9 \mathrm{cmH}_{2} \mathrm{O}$, IPAP minimum 8-12 $\mathrm{cmH}_{2} \mathrm{O}$ and IPAP maximum 25 $\mathrm{cmH}_{2} \mathrm{O}$ with further settings as needed for patient comfort and effectiveness. This device auto-titrates the EPAP to control apneic events, and the IPAP to control hypopneas. Auto-titrating devices were chosen in an attempt to achieve adequate SDB control as quickly as possible. The patient/ device interface was based on the patient's preference and effectiveness (acceptable level of leak, $<25 \mathrm{~L} / \mathrm{min}$ ). Adherence data were obtained via device download at 3,6 , and 12 months. Overnight home $\mathrm{SpO}_{2} / \mathrm{tc}-\mathrm{pCO}_{2}$ monitoring was repeated at 3,6, and 12 months. Adherence was recorded for all participants who had a PAP device available for use (nights without using the device were recorded as "zero's", if they did not accept or lost access to their PAP device, in which case they were deemed to have withdrawn from adherence monitoring). Individuals without SDB were not prescribed a PAP device but completed symptom logs and questionnaires per protocol.

We certify that all applicable institutional and government regulations concerning the ethical use of human volunteers were followed during the course of this research.

\section{Statistical analysis}

The event log data were dichotomized based on whether 1 or more events were recorded for a given subject. The observations were paired by subject and the event rates were compared using homogeneity analysis methods based on the McNemar's test. Mean quality of life questionnaire scores were compared for each follow-up interval relative to baseline. The data are paired by individual, and paired 


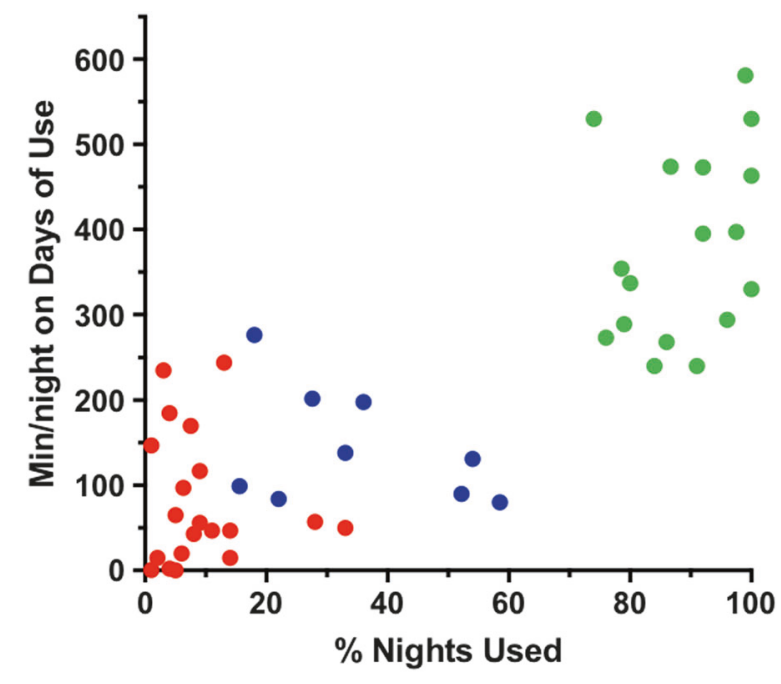

Fig. 2 Positive airway pressure (PAP) patterns of use. Each data point represents PAP use (\% nights used and minutes per night on nights used) for an individual participant, as determined by device downloads at the Month 3 time point. High-level users ( $\geq 70 \%$ nights used and $\geq 240$ min per night) are indicated in green. Medium-level users $(\geq 15 \%$ nights used and $\geq 60 \mathrm{~min}$ per night) in blue and low-level users $(<15 \%$ nights used or $<60$ min per night) in red

Student $t$-tests were used to assess for a difference in means. For analysis of factors predicting device use, either the percentage of days used or the number of minutes per night on nights of use were used as dependent variables in linear regression models. Clinical variables were assessed together, regardless of device type (BiPAP-auto vs. BiPAP/ AVAPS), as we showed previously that the presence or absence of nocturnal hypercapnia was not predicted by these factors [1]. Because BiPAP-AVAPS and BiPAP-auto titrate settings according to different algorithms, the predictive value of the device parameters (IPAP, EPAP, exhaled tidal volume (Vte) were examined separately with device type-specific models. All analyses were performed using $\mathrm{R}$ version 3.1 software.

\section{Results}

\section{Clinical features and SDB}

Of 252 participants contacted, 91 eligible participants agreed to participate; 75 male and 16 female participants, age $48 \pm 12$ years (mean $\pm S D$, range: $20-75$ years) enrolled. Participants were $17 \pm 12$ years post-injury (mean \pm SD, range: 1-50 years). The distribution of SCI motor levels and American Spinal Injury Association (ASIA) classification are detailed in our previous publication [1] but can be summarized as follows: $\mathrm{C} 1-3,4 \%$; $\mathrm{C} 4-6,59 \%$; $\mathrm{C} 7-8,12 \%$; T-level 26\%. Seventy-four individuals underwent HSAT and $\mathrm{SpO}_{2} / \mathrm{tc}-\mathrm{pCO}_{2}$ testing. The results of these studies were presented in detail in ref. [1]. To summarize, $81 \%$ had evidence of obstructive sleep apnea (OSA; $\geq 10 \mathrm{~s}$ periods of absent nasal airflow associated with stable or increasing chest/abdominal movement). Of the abnormal studies, there were a median of 12.4 obstructive events per hour (interquartile range (IQR): 9-25.5 events per hour; range: 5.1-60 events per hour). In $50 \%$ of the studies, the OSA was mild (5-15 events per hour) and in $50 \%$ it was moderate or severe ( $>15$ events per hour). Hypercapnea $\left(\mathrm{pCO}_{2} \geq 50\right.$ $\mathrm{mmHg}$ for $>5 \%$ of the recording time) was found in $28 \%$ of the studies. In the abnormal studies, hypercapnia was present for $25 \%$ of the study time (median; IQR: 11-72; maximum $100 \%$ ) and the median maximum $\mathrm{pCO}_{2}$ was 57 mmHg (IQR: 54-61 mmHg; maximum $95 \mathrm{mmHg}$ ) [1].

\section{PAP device use}

After 3 months, device downloads and $\mathrm{SpO}_{2} / \mathrm{tc}-\mathrm{pCO}_{2}$ results were analyzed. Both the consistency and duration of PAP therapy varied markedly, as shown in the scatter plot of $\%$ nights of device use vs. minutes per night on nights used (Fig. 2). As a further expression of this diversity, we arbitrary defined high-level use as $\geq 70 \%$ nights used and $\geq 240$ min per night, conforming with CMS adherence standards used in the US and most other insurance providers. Medium-level use was arbitrarily defined as $\geq 15 \%$ nights used and $\geq 60$ min per night, but less than high-level criteria. Lastly, low-level use was defined by $<15 \%$ nights used or $<60 \mathrm{~min}$ per night. By these criteria, at month 3 , $38 \%$ of participants were high-level users $(87 \pm 12 \%$ nights, $374 \pm 115$ min per night; mean \pm SD), $20 \%$ were mediumlevel users $(35 \pm 16 \%$ nights, $144 \pm 68 \mathrm{~min}$ per night), and $42 \%$ were low-level users $(9 \pm 9 \%$ nights, $85 \pm 77$ min per night). As shown in Fig. 2, the medium-level users were a mixed group of participants that used PAP fairly regularly but for limited duration, as well as some who used PAP for longer durations but only sporadically. PAP use was generally consistent over time; of 17 participants with highlevel use at month 3, 12 (70.6\%) continued high-level use throughout the study, whereas 5 (29.4\%) high-level users later used PAP at a lower level. Two participants converted from medium-level or low-level use at month 3 to highlevel use at month 6 or 12. These later results could be skewed, however, as participants who withdrew from the study after month 3 were mostly low-level users (not shown).

After 3 months, 27 individuals were using BiPAP-Auto and 16 had an apnea-hypopnea index (AHI) of $<5$ events per hour. In the $11(40.7 \%)$ with an AHI $>5$ events per hour at 3 months, only 3 had an obstructive apnea index of $>5$ events per hour. Therefore, BiPAP-Auto was effective in treating OSA in $89 \%$ of participants. There was residual central apnea in four $(15 \%)$ individuals at month 3. Overall, 
Table 1 Linear regression analysis of device settings as predictors for PAP device use at month 3

\begin{tabular}{|c|c|c|c|c|}
\hline Device & Predictor variable & Coefficient $^{\mathrm{a}}$ & Standard error & $p$ \\
\hline \multicolumn{5}{|l|}{$\%$ Days of device use } \\
\hline \multirow[t]{2}{*}{ BiPAP-auto $\left(R^{2} 0.19\right)$} & Average EPAP & 10.01 & 4.59 & $\mathbf{0 . 0 3 9}$ \\
\hline & Average IPAP & -2.78 & 4.06 & 0.5 \\
\hline \multicolumn{5}{|l|}{ BiPAP/AVAPS $\left(R^{2} 0.50\right)$} \\
\hline & Average EPAP & 12.23 & 5.871 & 0.059 \\
\hline & Average IPAP & 4.67 & 2.12 & 0.048 \\
\hline & Average Vte & 0.052 & 0.034 & 0.159 \\
\hline \multicolumn{5}{|l|}{ Minutes of use per night } \\
\hline \multirow[t]{2}{*}{ BiPAP-auto $\left(R^{2} 0.24\right)$} & Average EPAP & 42.12 & 19.43 & 0.04 \\
\hline & Average IPAP & -2.68 & 17.18 & 0.88 \\
\hline \multirow[t]{3}{*}{ BiPAP/AVAPS $\left(R^{2} 0.48\right)$} & Average EPAP & 12.23 & 5.87 & 0.059 \\
\hline & Average IPAP & 4.67 & 2.12 & 0.048 \\
\hline & Average Vte & 0.052 & 0.034 & 0.159 \\
\hline
\end{tabular}

a The coefficient refers to the unit change in the dependent variable for every unit change in the independent variable, based on the prediction model; for BiPAP-auto, the coefficient refers to the unit change in use (\% of days used or minutes per night on days used, as indicated) per $\mathrm{cmH}_{2} \mathrm{O}$ change in pressure. For BiPAP-AVAPS, the coefficient refers to the unit change in use (\% of IPAP or EPAP days used or minutes per night on days used, as indicated) per $\mathrm{cmH}_{2} \mathrm{O}$ change in pressure (IPAP or EPAP), or per L/min change in Vte, as indicated. Bold values indicate $p<0.05$

at month 3 , there were $3.2 \pm 3.9$ central events per hour. There was only one subject that required a change in device, and that occurred in the first 3 months of treatment. BiPAPAuto did not worsen central apnea in the majority of participants, as there were only two with increased central events, and under 3 events per hour.

At 3 months, 17 individuals were using BiPAP-AVAPS for $\mathrm{NH} \pm \mathrm{OSA} / \mathrm{CSA}$. A majority $13(77 \%)$ had improvement in the AHI, $6(35 \%)$ with an $\mathrm{AHI}<5$. Nocturnal hypercapnea completely resolved in $8(47 \%)$ and another 5 $(29 \%)$ had some improvement, whereas $1(6 \%)$ had worsening NH (3 did not complete testing).

\section{Prediction of PAP device use}

To determine whether there were predictors for PAP device adherence, we examined SCI motor level, severity of SDB at baseline, device type and device settings. Over the first 3 months of PAP use, the percent of days the device was used (\% days used) or minutes per night (min per night) of use on days used were not predicted by the SCI level (correlation coefficient $-1.2, p=0.7$ ), or a composite index of SDB severity (sum of obstructive, central, and hypopnea events; correlation coefficient 0.1 , $p=0.8$ ) [1]. In addition, individuals prescribed BiPAPAuto were no more or less likely to be adherent with therapy than those prescribed BiPAP-AVAPS. Mask type (nasal or nasal pillows versus nasal/oral or total face mask) also did not predict either \% days used or minutes per night of PAP use.
At month 3, in individuals prescribed BiPAP-Auto, average EPAP had significant predictive value for both $\%$ days used and minutes per night $(p=0.04)$ and $\%$ days used $(p=0.04)$. For individuals prescribed BiPAP/AVAPS, average IPAP had significant predictive value for both minutes per night used $(p=0.014)$ and $\%$ days used $(p=$ 0.048) (Table 1).

\section{Outcomes}

Daily event $\log$ completion rates were $96 \%$ at month 0 and $84 \%$ at month 3 . In an intention to treat analysis, participants at 6 months reported significant reductions in the frequency of symptoms suggesting autonomic dysreflexia (flushing, piloerection, nasal congestion, headache, or sweating), $p=0.01$ and orthostatic hypotension (lightheadedness or dizziness when sitting up or changing position) $p=0.01$ compared to month 0 (Fig. 3). The frequency of days with pulmonary symptoms (requiring more than usual treatments to clear secretions, or an increase in oxygen use) decreased significantly at 12 months, $p=0.04$ (Fig. 3). Unfortunately, there could be no valid comparison to the 11 participants without SDB, as they had very few of these events at baseline. Although improvements in these adverse events were not isolated to participants with highlevel use, the sizes of the cohorts with each level of use, and the frequencies of the adverse events, were insufficient to determine if any threshold level of PAP use was necessary to improve these outcomes. Larger cohorts in each user group would be necessary to describe and relationship between PAP use and effects on these outcomes. 
A
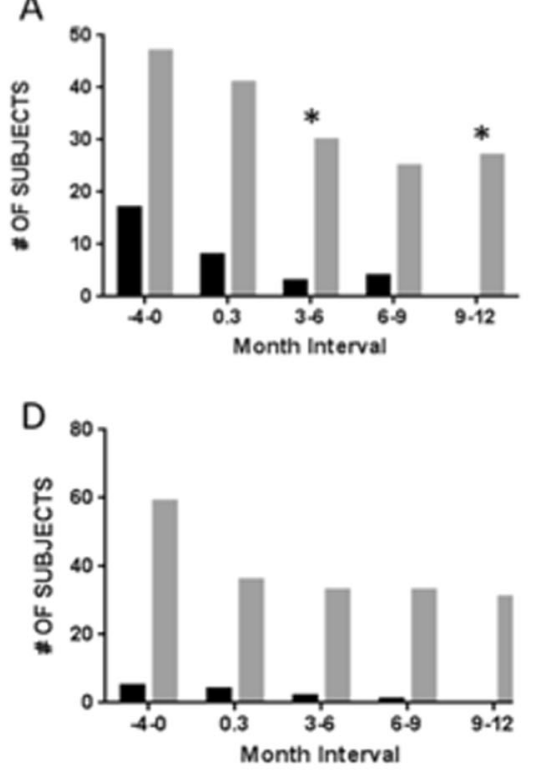

B

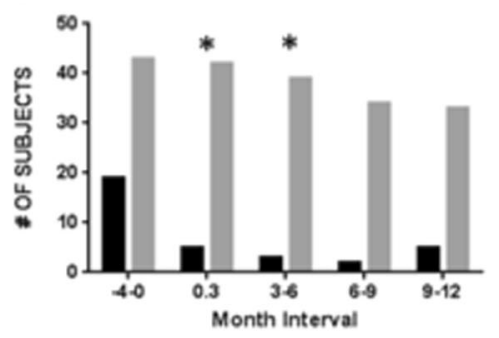

$E$

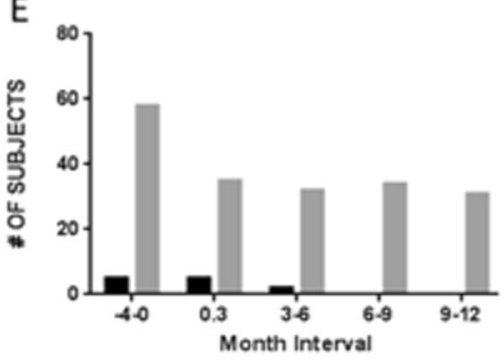

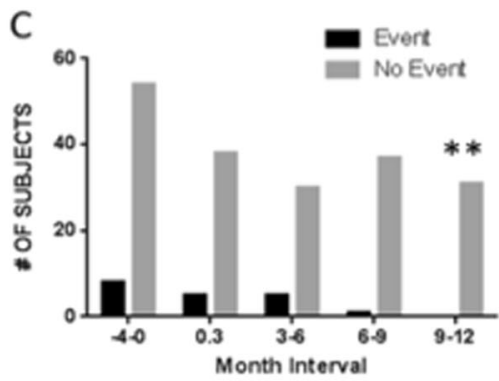

$\mathrm{F}$

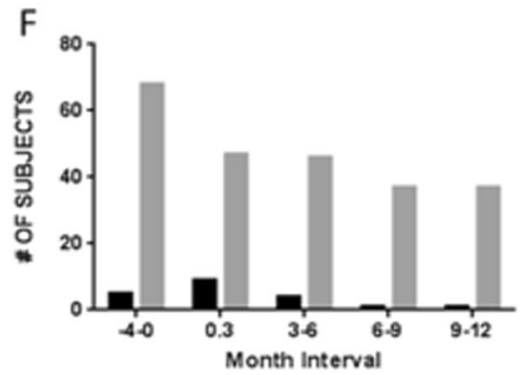

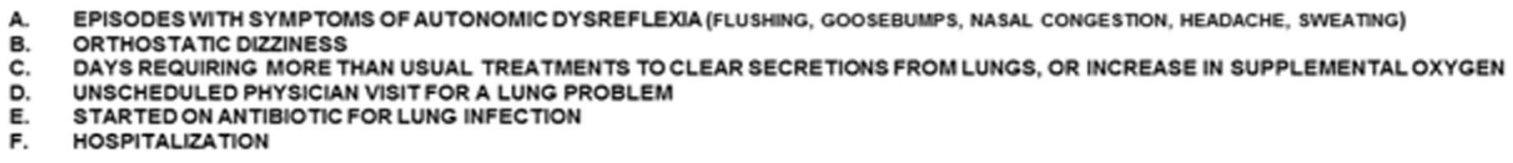

ALL STATISTICAL COMPARISONS ARE TO MONTHINTERVAL -4.0 (SEE METHODS). " p< $0.02 ; * * 0<0.05$

Fig. 3 Events recorded in participant daily logs. Each graph demonstrates the $n$ of participants recording at least one event or no events during the indicated time interval

Participants were also asked to complete the SF-12v.2, Brief Pain Inventory-SF (BPI), and Epworth Sleepiness Scale (ESS) quality of life surveys at enrollment and at 3,6, and 12 months (Appendix Table 4). Although the changes in QOL were modest, several were statistically significant over time (Table 2). The level of PAP use could not be related to QOL outcomes, in part due to the many subject withdrawals at later time points (not shown). The 11 individuals without SDB were also asked to complete surveys per study protocol. In general, there were not significant changes in responses, with two exceptions as shown in Table 2. Because so many participants left the study prematurely, and so many factors unrelated to PAP therapy vary widely from day to day (comorbidities, medications), this QOL analysis should be considered exploratory and future studies will be required to ascertain what benefits can be attributed specifically to PAP therapy, and the clinical contexts in which these benefits may be accrued.

\section{Discussion}

In this study, we evaluated bi-level PAP therapy for treatment of SDB in individuals with SCI. The risk of SDB after spinal cord injury is much higher than that of the able- bodied general population [1-6, 9]. Factors such as level of motor deficit, neck circumference, abdominal girth, respiratory muscle weakness, reduced ventilatory drive during sleep, analgesics, and other sedating medications may contribute to the high prevalence $[1,2,5]$. Given that OSA is a risk factor for motor vehicle accidents, which cause a significant number of SCI in the US, selection bias is also possible [29, 32].

We have recently shown that unsupervised, home based sleep apnea testing with transcutaneous capnography is an effective and practical approach for diagnosing SDB in individuals with SCI [1]. This study reports the treatment and follow-up of this cohort. Adherence with PAP therapy both in the general population and in individuals with SCI is low [18-21, 29]. In many cases, SCI patients refuse to even trial PAP therapy because they perceive that it will further complicate their care, they are concerned that the PAP interface would limit communication with caregivers, or they are unconvinced of current and future benefit from using PAP therapy. In this study, the majority of participants diagnosed with SDB agreed to initiate PAP therapy. After 3 months, 38\% used PAP at levels that would conform to CMS standards, which is similar to prior reports [19]. In addition, there were $20 \%$, arbitrarily defined as "medium users", whose PAP use was arguably meaningful, 
Table 2 Quality of life survey responses (from Appendix Table 3) (data collected as integers corresponding to each response, as shown)

Respondents qualifying for PAP therapy

Question 1: In general, would you say your health is:

1: Poor, 2: fair, 3: good, 4: very good, 5: excellent

Mean month 0 score 3.3, increasing to 3.6 at month 12 ( $p=$ 0.048). Months 3 and 6, no significant change

Question 5: During the last 4 weeks, how much did pain interfere with your normal work:

1: All the time, 2: most of the time, 3 : some of the time, $4:$ a little of the time, 5: none of the time

Mean month 0 score 3.8 , increasing to 4 at month $3(p=0.03)$. Months 6 and 12, no significant change

Question 6: Have you felt downhearted and depressed during the past 4 weeks:

1: Not at all, 2: a little bit, 3: moderately, 4: quite a bit, 5: extremely

Mean month 0 score 2.8 , decreasing to 2.4 at month 3 ( $p<$ $0.001)$ and 2.4 at month $6(p<0.01)$. Months 12 , no significant change

Epworth Sleepiness Scale (total points)

Mean month 0 score 8.3, decreasing to 6.8 at month $12(p<$ 0.01 ). Months 6 and 12, no significant change

Respondents without sleep-disordered breathing

Question 13: on a scale from 0 (no pain) to 10 (bad as you can imagine, rank how much pain you have right now:

Mean month 0 score 3.9, decreasing to 3.0 at month 3 ( $p=$ 0.045). Months 6 and 12, no significant change

Epworth Sleepiness Scale (total points)

Mean month 0 score 9.4 , decreasing to 5.9 at month 3 ( $p=$ 0.007). Months 6 and 12, no significant change

Only questions with significant changes from month 0 at $\geq 1$ time point are shown

but because of either frequency or duration of use would not meet CMS criteria as "adherent" to treatment. The remaining $42 \%$ used PAP so inconsistently and sparingly that it would be difficult to characterize their treatment as clinically meaningful.

This is the first study in SCI where objective data from device downloads were used to assess PAP use and effectiveness of treatment, as opposed to patient history or survey information. The severity of SDB, SCI motor level, mask type, and device type had no significant predictive value. Our data suggest that there may be differences in adherence that are a function of device-specific settings (Table 1). In individuals prescribed BiPAP-Auto, the average EPAP was a significant predictor of device use, as a higher EPAP resulted in increased use (both \% of nights of use and minutes of use per night). Further, for individuals using BiPAP/AVAPS, a higher level of IPAP correlated with increased use. This was somewhat surprising as clinicians often assume that higher pressures have a negative impact on adherence. It is possible that the higher pressure settings were, in fact, more comfortable, improved sleep quality, or provided unintended feedback to the patient that the treatment was more therapeutic. The auto-titrating algorithms were highly successful overall, so higher pressures did not select a subgroup with more effective treatment. In retrospect, we suspect that in future studies, \% days used as a parameter of adherence may require somewhat different treatment than minutes per night on days of use. Many participants reported that they would discontinue PAP treatment for significant periods when they had unrelated illnesses and they often expressed the opinion that PAP was only tolerable when their clinical status was relatively stable and that minutes per night was a function of overall sleep quality, comfort, or perceived benefit. Although many important questions remain, these findings show that adherence with PAP is significantly less than suggested by patient-reported data. In subsequent studies, it may be necessary to collect detailed survey information on a daily basis to ascertain what determined the level of use for the previous night. Obtaining EEG data could also be used to further determine the effect on sleep quality in this patient population. Given the effectiveness of PAP therapy for SDB, future studies to inform how best to ensure adherence will be paramount.

Individuals with SCI have unique medical comorbidities, including symptoms of autonomic dysreflexia, orthostatic hypotension, impaired cough strength, and increased risk of pulmonary infections/pneumonia and pain. In this study, we sought to determine whether treatment of SDB has shortterm benefits toward these medical comorbidities, symptoms, and quality of life as prior studies have not addressed this issue. SDB has been associated with increased sympathetic activity, which plausibly could impact autonomic stability, specifically clinically significant blood pressure fluctuations [33, 34]. Notably, we observed substantial reductions in the frequency of autonomic dysreflexia symptoms and orthostatic dizziness at several time points (Fig. 3). In addition, there was a delayed improvement in the frequency of days with increased pulmonary symptoms, whereas reductions in unscheduled physician visits, respiratory infections, and hospitalizations did not change significantly. This may be partly due to the relatively low frequencies of these events, compared to the symptoms of blood pressure instability, so any benefits in these areas would require a larger study. To our knowledge, this is the first evidence to suggest that SDB treatment may favorably impact AD reactions or postural dizziness in SCI patients. Our findings, although provocative, must be substantiated by further studies where larger cohorts of participants remain for the entire study protocol and outcomes can be compared to those of participants without SDB. A relationship between $\mathrm{SDB}$ and $\mathrm{AD}$ reactions or postural 
Table 3 SF-12v.2 Health Survey

UNIVERSITY OF MICHIGAN

SPINAL CORD INJURY

RESEARCH PROJECT

Name

Date

\section{SF-12v.2 HEALTH SURVEY}

This survey asks for your views about your health. This information will help keep track of how you feel and how well you are able to do your usual activities.

Answer every question by selecting the answer as indicated. If you are unsure about how to answer a question, please give the best answer you can.

1. In general, would you say your health is:

$\begin{array}{ccccc}\text { Excellent } & \text { Very good } & \text { Good } & \text { Fair } & \text { Poor } \\ \bigcirc & 0 & \bigcirc & \bigcirc & 0\end{array}$

2. The following questions are about activities you might do during a typical day. Does your health now limit you in these activities? If so, how much?

not

Yes, Yes, No,

limited limited

limited

a lot a little at all

a. Moderate activities, such as moving a table, pushing a

vacuum cleaner, bowling, or playing golf

$0 \quad 0 \quad 0$

b. Climbing several flights of stairs

3. During the past 4 weeks, how much of the time have you had any of the following problems with your work or other regular daily activities as a result of your physical health?

\begin{tabular}{|c|c|c|c|}
\hline $\begin{array}{l}\text { All } \\
\text { f the }\end{array}$ & $\begin{array}{l}\text { Most } \\
\text { of the } \\
\text { time }\end{array}$ & $\begin{array}{l}\text { Some } \\
\text { of the } \\
\text { time }\end{array}$ & $\begin{array}{c}\text { A little } \\
\text { of the } \\
\text { time }\end{array}$ \\
\hline
\end{tabular}

a. Accomplished less than you would like

$0 \quad 0 \quad 0$

b. Were limited in the kind of work or other activities.

4. During the past 4 weeks, how much of the time have you had any of the following problems with your work or other regular daily activities as a result of any emotional problems (such as feeling depressed or anxious)?

\begin{tabular}{|c|c|c|c|}
\hline $\begin{array}{l}\text { All } \\
\text { of the } \\
\text { time }\end{array}$ & $\begin{array}{l}\text { Most } \\
\text { of the } \\
\text { time }\end{array}$ & $\begin{array}{l}\text { Some } \\
\text { of the } \\
\text { time }\end{array}$ & $\begin{array}{l}\text { A little } \\
\text { of the } \\
\text { time }\end{array}$ \\
\hline
\end{tabular}

a. Accomplished less than you would like

$0 \quad 0 \quad 0 \quad 0 \quad 0$

b. Did work or activities less carefully than usual 
Table 3 (continued)

5. During the past 4 weeks, how much did pain interfere with your normal work (including both work outside the home and housework)?

Not at all

A little bit

Moderately

Quite a bit

Extremely

$\bigcirc$
O

$\bigcirc$

$\bigcirc$

O

6. These questions are about how you feel and how things have been with you during the past 4 weeks. For each question, please give the one answer that comes closest to the way you have been feeling.

How much of the time during the past 4 weeks...

a. Have you felt calm and peaceful?

$\begin{array}{ccccc}\text { All } & \text { Most } & \text { Some } & \text { A little } & \text { None } \\ \text { of the of the } & \begin{array}{l}\text { of the of the } \\ \text { of the }\end{array} \\ \text { time } & \text { time } & \text { time time time }\end{array}$

b. Did you have a lot of energy?

c. Have you felt downhearted and depressed?

7. During the past 4 weeks, how much of the time has your physical health or emotional problems interfered with your social activities (like visiting friends, relatives, etc.)?

All

of the time
Most

of the time
Some

of the time
A little

of the time
None

of the time

$\bigcirc$

$\bigcirc$

$\bigcirc$

$\bigcirc$

\section{SUPPLEMENTAL QUESTIONS}

8. How many hours per week do you spend working at job for which you paid?

9. How many hours per week do you spend in homemaking activities, including parenting, meal preparation, and home maintenance?

10. How many hours per week do you spend in school, including both hours of class and time spent studying?

11. How many hours per week do you spend in recreational activities such as sports, exercising, going to movies, or playing games - do not include watching TV?

12. Do you live with a spouse or significant other?

Yes

No

(check one) 


\section{QUESTIONS FROM THE BRIEF PAIN INVENTORY}

13) On a scale from 0 to 10, with 0 being No Pain and 10 being Pain as Bad as You Can Imagine, please rank how much pain you have right now

14) On a scale from 0 to 10, with 0 being Pain Does Not Interfere and 10 being Pain Completely Interferes, how much has your pain interfered with your General Activities during the past $\mathbf{2 4}$ hours?

\section{EPWORTH SLEEPINESS SCALE}

How likely are you to doze off or fall asleep in the situations described below, in contrast to feeling just tired?

This refers to your usual way of life in recent times.

Even if you haven't done some of these things recently, try to work out how they would have affected you.

Use the following scale to choose the most appropriate number for each situation.

$$
\begin{aligned}
& 0=\text { would never doze } \\
& 1=\text { Slight chance of dozing } \\
& 2=\text { Moderate chance of dozing } \\
& 3=\text { High chance of dozing }
\end{aligned}
$$

\section{Situation}

Chance of dozing

15. Sitting and reading.

16. Watching TV

17. Sitting, inactive in a public place (e.g. a theatre or a meeting)

18. As a passenger in a car for an hour without a break

19. Lying down to rest in the afternoon when circumstances permit

20. Sitting and talking to someone

21. Sitting quietly after a lunch without alcohol

22. In a car, while stopped for a few minutes in the traffic 
Table 4 Symptom/event log

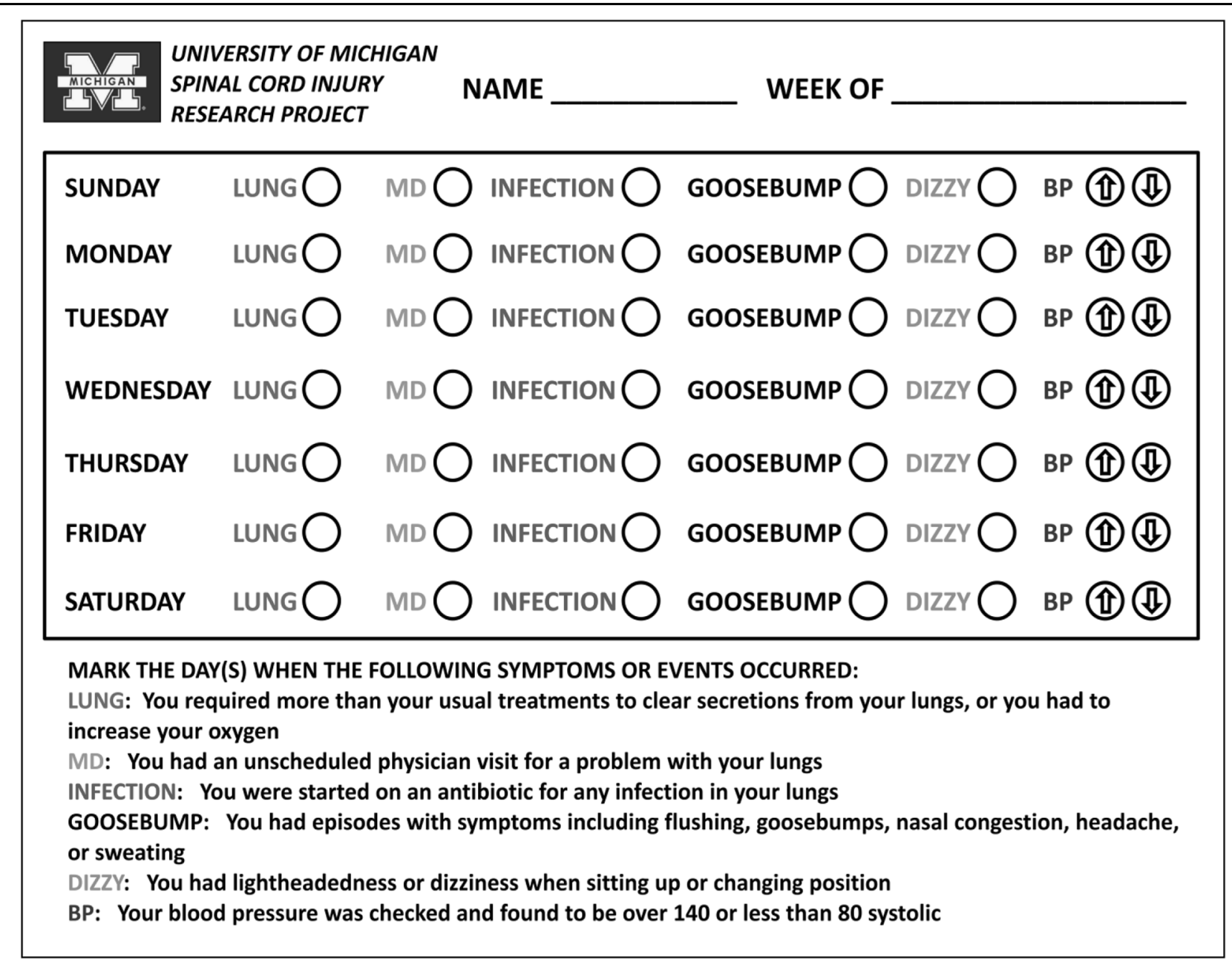

dizziness is entirely plausible, as SDB is associated with sympathetic hyperactivity and is a risk factor for hypertension in the general populations [33, 34].

We administered three quality of life surveys (Table 2; Appendix Table 4). Despite the suboptimal and variable device use from night to night, several areas of improvement were suggested in quality of life on an intention-totreat basis. Although there were few patients with no SDB for comparison, our data suggest that there may be shortterm benefits to be gained from PAP use. Like the event log results, it is perhaps not surprising that some of these benefits were not consistent throughout the follow-up period, given the variations in device use, the influence of common concurrent illnesses such as urinary tract or wound infections, and frequent changes in medications. Future studies comparing such symptoms between those who are highly adherent with PAP use and those who either refuse or who have low PAP adherence would also serve to strengthen our findings.

\section{Study limitations}

A significant limitation of this study is the high rate of participants voluntarily leaving the study. The study started with 91 participants and at the end of 12 months only 37 were still enrolled. In most instances, we were able to use paired-data analyses, so the loss of participants during follow-up did not skew the results indicating short-term benefits, but the progressive reduction of the number of participants remaining in the study limited the power of the data analysis. In addition, because of the high prevalence of SDB in individuals with SCI, the control group was very small. Only 11 participants had no SDB, and 4 of these left the study after month 3 . For this reason, it is difficult to attribute any possible benefits to PAP therapy, as opposed to unintended consequences of study participation or other variables. Given the very high prevalence of SDB after SCI, any future study would have to have an initial enrollment 
many times larger than ours to overcome these limitations. Other limitations are the widely diverse patterns of PAP use and the common confounding effects of multiple comorbidities. However, data from studies such as ours may still inform future studies to target important determinants of day to day use of PAP therapy. Until these data are obtained, we can only speculate whether current criteria for treatment adherence adequately reflect the amount of device use necessary to confer meaningful clinical benefits.

\section{Conclusion}

Positive airway pressure therapy may have short-term benefits with regard to quality of life and blood pressure stability for individuals with SCI and SDB despite widely ranging frequencies and durations of use. Although a much larger patient cohort will be necessary to determine with more precision the levels of PAP treatment necessary to confer specific clinical benefits, our data raise the possibility that conventional standards for adherence to PAP therapy may be inappropriately rigorous, and that PAP may be recommended to patients with $\mathrm{SCI}$ as beneficial even if they are only able to accept treatment sporadically or for shorter durations.

Acknowledgements We wish to thank Kerby Shedden, Ph.D., Director for the University of Michigan Center for Statistical Analysis and Consultation and Research for his advice and expertise. We would also would like to thank Marty Frick and Katie Gootee for administrative support.

\section{Compliance with ethical standards}

Conflict of interest The authors declare that they have no conflict of interest.

\section{References}

1. Bauman KA, Kurili A, Schotland HM, Rodriguez GA, Chiodo AE, Sitrin RG. A simplified approach to diagnosing sleep-disordered breathing and nocturnal hypercapnia in individuals with spinal cord injury. Arch Phys Med Rehabil. 2016;97:363-71.

2. Berlowitz DJ, Brown DJ, Campbell DA, Pierce RJ. A longitudinal evaluation of sleep and breathing in the first year after cervical spinal cord injury. Arch Phys Med Rehabil. 2005;86:1193-9.

3. Burns SP, Little JW, Hussey JD, Lyman P, Lakshminarayanan S. Sleep apnea syndrome in chronic spinal cord injury: associated factors and treatment. Arch Phys Med Rehabil. 2000;81:1334-9.

4. Klefbeck B, Sternhag M, Weinberg J, Levi R, Hultling C, Borg J. Obstructive sleep apneas in relation to severity of cervical spinal cord injury. Spinal Cord. 1998;36:621-8.

5. Leduc BE, Dagher JH, Mayer P, Bellemare F, Lepage Y. Estimated prevalence of obstructive sleep apnea-hypopnea syndrome after cervical cord injury. Arch Phys Med Rehabil. 2007;88:333-7.
6. Short DJ, Stradling JR, Williams SJ. Prevalence of sleep apnoea in patients over 40 years of age with spinal cord lesions. J Neurol Neurosurg Psychiatr. 1992;55:1032-6.

7. Epstein LJ, Kristo D, Strollo PJ Jr, Friedman N, Malhotra A, Patil SP, et al. Clinical adults. J Clin Sleep Med. 2009;5:263-76.

8. Jennum P, Riha RL. Epidemiology of sleep apnoea/hypopnoea syndrome and sleep-disordered breathing. Eur Respir J. 2009;33:907-14.

9. Sankari A, Martin JL, Bascom AT, Mitchell MN, Badr MS. Identification and treatment of sleep-disordered breathing in chronic spinal cord injury. Spinal Cord. 2015;53:145-9.

10. Angelico F, del Ben M, Augelletti T, de Vita R, Roma R, Violi F, et al. Obstructive sleep apnoea syndrome and the metabolic syndrome in an internal medicine setting. Eur $\mathbf{J}$ Intern Med. 2010;21:191-5.

11. Botros N, Concato J, Mehsenin V, Selim B, Doctor K, Yaggi HK. Obstructive sleep apnea as a risk factor for type 2 diabetes. Am J Med. 2009;122:1122-7.

12. Buchner NJ, Sanner BM, Borgel J, Rump LC. Continuous positive airway pressure treatment of mild to moderate obstructive sleep apnea reduces cardiovascular risk. Am J Respir Crit Care Med. 2007;176:1274-80.

13. Levy P, Bonsignore MR, Eckel J. Sleep, sleep-disordered breathing and metabolic consequences. Eur Respir J. 2009;34:243-60.

14. Papanas N, Steiropoulos R, Nena E, Tzouvelekis A, Maltezos E, Trakada G, et al. HbA1c is associated with severity of obstructive sleep apnea-hypopnea syndrome in nondiabetic men. Vasc Health Risk Manag. 2009;5:751-6.

15. Parish JM, Adam T, Facchiano L. Relationship of metabolic syndrome and obstructive sleep apnea. J Clin Sleep Med. 2007;3:467-72.

16. Somers VK, White DP, Amin R, Abraham WT, Costa F, Culebras A, et al. Sleep apnea and cardiovascular disease: an American Heart Association/American College of Cardiology Foundation Scientific Statement from the American Heart Association Council for High Blood Pressure Research Professional Education Committee, Council on Clinical Cardiology, Stroke Council, and Council on Cardiovascular Nursing. J Am Coll Cardiol. 2008;52:686-717.

17. Vgontzas AN, Bixler EO, Chrousos GP. Sleep apnea is a manifestation of the metabolic syndrome. Sleep Med Rev. 2005;9:211-24.

18. Burns SP, Kapur V, Yin KS, Buhrer R. Factors associated with sleep apnea in men with spinal cord injury: a population-based case-control study. Spinal Cord. 2001;39:15-22.

19. Wickwire EM, Lettieri CJ, Cairns AA, Collop NA. Maximizing positive airway pressure adherence in adults: a common-sense approach. Chest. 2013;144:680-93.

20. Kribbs NB, Pack AI, Kline LR, Smith PL, Schwartz AR, Schubert NM, et al. Objective measurement of patterns of nasal CPAP use by patients with obstructive sleep apnea. Am Rev Respir Dis. 1993;147:887-95.

21. Sin DD, Mayers I, Man GC, Pawluk L. Long-term compliance rates to continuous positive airway pressure in obstructive sleep apnea: a population-based study. Chest. 2002;121:430-5.

22. Billings ME, Auckley D, Bence R, Foldvary-Schaefer N, Iber C, Redline $\mathrm{S}$, et al. Race and residential socioeconomics as predictors of CPAP adherence. Sleep. 2011;34:1653-8.

23. Chasens ER, Pack AI, Maislin G, Dinges DF, Weaver TE. Claustrophobia and adherence to CPAP treatment. West J Nurs Res. 2005;27:307-21.

24. El-Solh AA, Ayyar L, Akinnusi M, Relia S, Akinnusi O. Positive airway pressure adherence in veterans with post-traumatic stress disorder. Sleep. 2010;33:1495-1500. 
25. Engleman HM, Wild MR. Improving CPAP use by patients with the sleep apnoea/hypopnoea syndrome (SAHS). Sleep Med Rev. 2003;7:81-99.

26. Ballard RD, Gay PC, Strollo PJ. Interventions to improve compliance in sleep apnea patients previously non-compliant with continuous positive airway pressure. J Clin Sleep Med. 2007;3:706-12.

27. Ip S, D'Ambrosio C, Patel K, Obadan N, Kitsios GD, Chung M, et al. Auto-titrating versus fixed continuous positive airway pressure for the treatment of obstructive sleep apnea: a systematic review with meta-analyses. Syst Rev. 2012;1:20.

28. Smith I, Lasserson TJ. Pressure modification for improving usage of continuous positive airway pressure machines in adults with obstructive sleep apnoea. Cochrane Database Syst Rev. 2009; CD003531, https://doi.org/10.1002/14651858.CD003531.pub3.

29. Berlowitz DJ, Spong J, Pierce RJ, Ross J, Barnes M, Brown DJ. The feasibility of using auto-titrating continuous positive airway pressure to treat obstructive sleep apnoea after acute tetraplegia. Spinal Cord. 2009;47:868-73.

30. Bisogni V, Pengo MF, Maiolino G, Rossi GP. The sympathetic nervous system and catecholamines metabolism in obstructive sleep apnoea. J Thorac Dis. 2016;8:243-54.

31. Waring WP 3rd, Biering-Sorensen F, Burns S, Donovan W, Graves D, Jha A, et al. 2009 review and revisions of the international standards for the neurological classification of spinal cord injury. J Spinal Cord Med. 2010;33:346-52.

32. Ellen RL, Marshall SC, Palayew M, Molnar FJ, Wilson KG, Man-Son-Hing M. Systematic review of motor vehicle crash risk in persons with sleep apnea. J Clin Sleep Med. 2006;2:193-200.

33. Friedman O, Logan AG. Sympathoadrenal mechanisms in the pathogenesis of sleep apnea-related hypertension. Curr Hypertens Rep. 2009;11:212-6.

34. Narkiewicz K, Somers VK. Sympathetic nerve activity in obstructive sleep apnoea. Acta Physiol Scand. 2003;177:385-90. 